\title{
NOTE ON VECTOR FIELDS IN MANIFOLDS ${ }^{1}$
}

\author{
HANS SAMELSON
}

In memoriam Heinz Hopf, June 3, 1971

\begin{abstract}
We give a direct geometric proof of Hopf's theorem on the sum of indices at the zeros of a vector field in a manifold $M$, or rather of that part of the theorem that says that the sum is the same for any two vector fields. The main idea is to connect the two fields by a one-parameter family of fields and to make everything transversal (to $M \times I$ ). The resulting system of curves permits one to read off the theorem.
\end{abstract}

The classical theorem of $\mathrm{H}$. Hopf on vector fields in a manifold [2] (see also Lefschetz [3]) can be stated as follows: Let $M$ be a differentiable (say $C^{\infty}$ ) compact connected closed manifold, and let $V$ be a (differentiable) vector field in $M$ with a finite number of zeros; then the sum of the indices of the zeros of $V$ equals the Euler characteristic of $M$. We propose to give a short proof of that part of the theorem that says that the sum of the indices of the zeros is the same for any two vector fields. (That the common value is the Euler characteristic of $M$ can then be seen by taking for $V$ the "gradient field" of a Morse function.) The main tool will be transversality ("general position") ([1], [4], [5]). We take as known the definition and basic properties of the index of a zero: in any coordinate system, with the zero as origin, $V$ defines a map of the unit sphere into itself; the index is the degree of this map. If we deform $V$ slightly to $V^{\prime}$, then any zero of $V^{\prime}$ must appear near a zero of $V$, and the index of any zero of $V$ equals the sum of the indices of the nearby zeros of $V^{\prime}$.

We regard a vector field as a section of the tangent bundle $T M$ of $M$. The transversality theorem ([1], [4], [5]) and the last remark above allow us to restrict ourselves to transversal vector fields, that is, vector fields that are transversal to the 0 -section of $T M$ (which we identify with $M$ itself). For such a vector field the zeros are automatically isolated;

Received by the editors January 10, 1972 and, in revised form, March 24, 1972.

AMS 1969 subject classifications. Primary 5734, 5732.

Key words and phrases. Manifold, vector field, index, transversality.

${ }^{1}$ Work supported in part by NSF grant GP-29696.

(c) American Mathematical Society 1972 
moreover, each index is +1 or -1 : with $V$ considered as a submanifold of $T M$, the tangent space $V_{p}$ to $V$ at a zero $p$ can be regarded as graph of a linear map from $M_{p}$, the (horizontal) tangent space of the 0 -section $M$ of $T M$ at $p$, to $M_{p}$, the (vertical) tangent space to the fiber $M_{p}$ of $T M$ at 0 [with the usual convention about the tangent space of a vector space]. Transversality insures, or even means, that this map from $M_{p}$ to itself is nonsingular; the index is the sign of the determinant; it is $+1(-1)$ if the orientation is preserved (reversed).

Suppose now that $V$ and $W$ are two transversal vector fields. We consider the manifold $T M \times I$; it is a vector bundle over $M \times I$, with 0 -section $M \times I$ and fiber $M_{p}$ at $(p, t)$. The fields $V$ and $W$ give a section of this bundle over the subset $M \times 0 \cup M \times 1$ of $M \times I$. We extend this to a continuous section $S$ over $M \times I$; e.g., for $0 \leqq t \leqq \frac{1}{3}$ we take $(1-3 t) \cdot V$, then 0 for $\frac{1}{3} \leqq t \leqq \frac{2}{3}$, and finally $(3 t-2) \cdot W$ for $\frac{2}{3} \leqq t \leqq 1$. Then, by the transversality theorem ([1], [3], [4]), we approximate $S$ by a differentiable section $Z$, which is transversal to $M \times I$ and agrees with $S$ on some $M \times$ $[0, \varepsilon] \cup M \times[1-\varepsilon, 1]$, where $S$ is already differentiable and transversal; $Z$ agrees with $V$ on $M \times 0$ and with $W$ on $M \times 1$. By the main property of transversality the intersection of $Z$ (regarded as a submanifold of $T M \times I$ ) and $M \times I$ is of dimension 1 , and consists of a finite number of disjoint closed curves (in $M \times(0,1)$ ) and arcs (whose endpoints are on $M \times 0 \cup$ $M \times 1)$. The endpoints of the arcs are precisely the zeros of $V$ and $W$. Suppose such an arc, $\gamma$, has both endpoints, $(a, 0)$ and $(b, 0)$, on $M \times 0$. Then one sees easily, by moving along $\gamma$, that of the indices of $V$ at $a$ and $b$ one is +1 and the other -1 : First we note that the tangent space to $T M \times I$ at a point $(p, t)$ of $M \times I$ is of the form $M_{p}$ (horizontal) $\oplus M_{p}$ (vertical) $\oplus R$, and so has a canonical orientation. We take a frame at $(a, 0)$, consisting of $\dot{\gamma}$ (tangent to $\gamma$ ), horizontal vectors $X_{1}, \cdots, X_{n}$ (a basis for $M_{a}$ ), and vertical vectors $Y_{1}, \cdots, Y_{n}$, images of the $X_{i}$ under the linear map with the tangent space to $V$ as graph, described above. We move this frame continuously along $\gamma$, always with $\dot{\gamma}$ as first vector, and always using the tangent space to $Z$ to map the horizontal $X_{i}$ to vertical $Y_{i}$; the $X_{i}$ at $(b, 0)$ should be in $M_{l}$. The fact that $\dot{\gamma}$ points into $M \times I$ at $(a, 0)$, but out of $M \times I$ at $(b, 0)$, means that the orientations of $M_{a} \oplus M_{a}$ and $M_{b} \oplus M_{b}$, defined by $X_{1}, \cdots, X_{n}, Y_{1}, \cdots, Y_{n}$, are opposite to each other. But that is equivalent to our claim about the indices of $V$ at $a$ and $b$. Similarly for arcs with both ends on $M \times 1$. Finally, if an arc goes across from $M \times 0$ to $M \times 1$, one sees by the same reasoning that the two endpoints have the same index, both +1 or both -1 . It is clear now that the sum of \pm 1 's over the zeros of $V$ equals that over the zeros of $W$ (the contributions of the endpoints of any arc that does not go "across" cancel); the theorem is proved. 


\section{REFERENCES}

1. R. Abraham and J. Robbin, Transversal mappings and flows, Benjamin, New York, 1967, p. 48. MR 39 \#2181.

2. H. Hopf, Vektorfelder in n-dimensionalen Mannigfaltigkeiten, Math. Ann. 96 (1926), 225-250.

3. S. Lefschetz, Continuous transformations of manifolds, Proc. Nat. Acad. Sci. U.S.A. 11 (1925), 290-292.

4. J. Milnor, Differential topology, Lecture Notes, Princeton University, Princeton, N.J., 1959, Theorem 1.35, p. 22.

5. R. Thom, Quelques propriétés globales des variétés différentiables, Comment. Math. Helv. 28 (1954), 17-86, Theorem I.5. MR 15, 890.

Department of Mathematics, Stanford University, Stanford, California 94305 